\title{
Uso Atual de Ressonância Magnética Cardíaca Pediátrica no Brasil
}

\section{Current Use of Pediatric Cardiac Magnetic Resonance Imaging in Brazil}

\author{
Marcelo Felipe Kozak, ${ }^{1,2}$ Jorge Yussef Afiune, ${ }^{1 \oplus}$ Lars Grosse-Wortmann ${ }^{3,4}$ \\ Instituto de Cardiologia do Distrito Federal - Cardiologia Pediátrica, ${ }^{1}$ Brasília, DF - Brasil \\ Hospital da Criança de Brasília José de Alencar - Ecocardiografia, ${ }^{2}$ Brasília, DF - Brasil \\ Doernbecher Children's Hospital, Oregon Health and Science University - Division of Pediatric Cardiology, Department of Pediatrics, ${ }^{3}$ \\ Portland, Oregon - EUA \\ The Hospital for Sick Children, University of Toronto - Department of Pediatrics, ${ }^{4}$ Toronto, Ontario - Canadá
}

\section{Resumo}

Fundamento: Dados sobre o uso de ressonância magnética cardíaca (RMC) em crianças no Brasil são escassos.

Objetivos: Buscamos oferecer informações sobre as práticas atuais de RMC pediátricas no Brasil.

Métodos: Um questionário foi enviado a médicos solicitantes de RMC de todo o país, cobrindo informações sobre si próprios, sobre seus serviços de RMC, contexto clínico dos pacientes e sobre os obstáculos para a realização de RMC em crianças. Para a análise estatística, um p $<0,05$ bilateral foi considerado significativo.

Resultados: A pesquisa obteve 142 respostas. Foi relatado que a RMC está disponível para $79 \%$ dos respondentes, dos quais $52 \%$ raramente ou nunca a utilizam. As indicações mais comuns são cardiomiopatias (84\%), pós-operatório de correção de tetralogia de Fallot $(81 \%)$ e malformações do arco aórtico (53\%). A complexidade do exame se correlacionou à relação $\mathrm{RMC} /$ cirurgia $(\mathrm{Rho}=0,48, \mathrm{IC} 95 \%=0,32-0,62, \mathrm{p}<0,0001)$ e ao número de exames de $\mathrm{RMC}(\mathrm{Rho}=0,52, \mathrm{IC}$ $95 \%=0,38-0,64, p<0,0001)$. A complexidade da RMC esteve associada à sua realização por cardiologistas pediátricos (RC 2,04, IC 95\% 1,2-3,89, p < 0,01). Os principais obstáculos ao uso mais frequente de RMC foram o alto custo (65\%), a necessidade de sedação (60\%) e o número insuficiente de profissionais qualificados $(55 \%)$.

Conclusão: A RMC pediátrica não é usada frequentemente no Brasil. A presença de um cardiologista pediátrico a frente dos exames esteve associado ao uso de RMC em pacientes mais complexos. $\mathrm{O}$ treinamento de especialistas em RMC pediátrica e a educação dos médicos solicitantes são passos importantes na direção de um uso mais abrangente de RMC no Brasil. (Arq Bras Cardiol. 2021; 116(2):305-312)

Palavras-chave: Pediatria; Cardiopatias Congênitas/cirurgia; Técnicas e Procedimentos Diagnósticos; Imagem por Ressonância Magnética; Cateterismo Cardíaco.

\begin{abstract}
Background: Data on the use of cardiac magnetic resonance imaging (CMR) on children in Brazil is lacking

Objectives: This study sought to provide information on current pediatric CMR practices in Brazil.
\end{abstract}

Methods: A questionnaire was sent out to referring physicians around the country. It covered information on the respondents, their CMR practices, the clinical context of the patients, and barriers to CMR use among children. For statistical analysis, two-sided p $<0.05$ was considered significant.

Results: The survey received 142 replies. CMR was reported to be available to $79 \%$ of the respondents, of whom, 52\% rarely or never use CMR. The most common indications were found to be cardiomyopathies (84\%), status of post-tetralogy of Fallot repair (81\%), and aortic arch malformations (53\%). Exam complexity correlated with CMR-to-surgery ratio (Rho $=0.48,95 \% \mathrm{Cl}=0.32-0.62, p<0.0001)$ and with the number of CMR exams (Rho $=0.52,95 \% \mathrm{Cl}=0.38-0.64, p<0.0001)$. Further, a high CMR complexity score was associated with pediatric cardiologists conducting the exams (OR 2.14, 95\% Cl 1.2-3.89, $p<0.01$ ). The main barriers to a more frequent use of CMR were its high cost (65\%), the need for sedation (60\%), and an insufficient number of qualified professionals (55\%).

Conclusion: Pediatric CMR is not used frequently in Brazil. The presence of a pediatric cardiologist who can perform CMR exams is associated with CMR use on more complex patients. Training pediatric CMR specialists and educating referring providers are important steps toward a broader use of CMR in Brazil. (Arq Bras Cardiol. 2021; 116(2):305-312)

Keywords: Pediatrics; Heart Defects, Congenital/surgery; Diagnostic Techniques Procedures; Magnetic Resonance Imaging; Cardiac Catheterization. Full texts in English - http://www.arquivosonline.com.br

Correspondência: Marcelo Felipe Kozak •

Instituto de Cardiologia - Estrada Parque Contorno do Bosque, S/N. CEP 70658-700, SHCES, Brasília, DF - Brasil

E-mail: marcelo.f.kozak@icloud.com

Artigo recebido em 11/12/2019, revisado em 25/05/2020, aceito em 10/06/2020

DOI: https://doi.org/10.36660/abc.20190860 


\section{Artigo Original}

\section{Introdução}

A ressonância magnética cardíaca (RMC) é considerada o método padrão-ouro para a avaliação de volumes ventriculares, função sistólica e quantificação de fluxo in vivo. ${ }^{1-5}$ Também pode diagnosticar edema e fibrose com boa concordância com a histologia. ${ }^{6,7}$ Além disso, as imagens de RMC podem ser usadas como matriz para produzir modelos cardíacos tridimensionais, que podem ser usados para ensino, treinamento e planejamento pré-cirúrgico..$^{8,9}$ A combinação dessas qualidades fazem da RMC uma ferramenta poderosa no manejo de pacientes com cardiopatia congênita (CC).

Na última década, a RMC tem sido empregada rotineiramente nos principais centros de cardiologia pediátrica do mundo. Em alguns hospitais, o número de exames de RMC ultrapassa o número de cirurgias cardíacas: No Boston Children's Hospital, por exemplo, houve 1270 exames de RMC e 947 cirurgias em 2016/2017 (http://www. childrenshospital. org); no Texas Children's Hospital, houve 941 exames de RMC e 926 cirurgias em 2018 (https://www.texaschildrens.org); e no Hospital for Sick Children em Toronto, aproximadamente 700 exames de RMC e 600 cirurgias são realizados por ano. Esses números se traduzem em uma relação RMC/cirurgia entre 1,02 e 1,34.

No Brasil, números sobre a utilização de RMC não estão disponíveis para consulta pública. A hipótese deste estudo é que a RMC não está amplamente disponível para a população pediátrica e que vem sendo subutilizada. Informações sobre seu uso, suas indicações mais frequentes e os obstáculos à sua utilização, bem como seu papel na tomada de decisões clínico-cirúrgicas, não estão disponíveis. Dados não publicados do Instituto de Cardiologia do Distrito Federal relatam que o número médio anual de exames de RMC pediátricos é de 55 , enquanto a média anual de cirurgias cardíacas pediátricas é de 180, resultando em uma relação RMC/cirurgia de 0,31. No Hospital da Criança e Maternidade de São José do Rio Preto, onde aproximadamente 300 cirurgias são realizadas todo ano, apenas 21 exames de RMC foram realizados em 2018 (comunicação pessoal), resultando em uma relação RMC/cirurgia de 0,07. Como esses números não são necessariamente representativos, buscamos obter informações sobre o uso de RMC em crianças em todo o Brasil.

\section{Métodos}

Uma pesquisa foi distribuída a cardiologistas pediátricos e cirurgiões cardíacos em todo o país. Esses indivíduos foram identificados, principalmente, a partir de três grupos de WhatsApp relacionados à área de cardiologia pediátrica: "Grande INCOR" (grupo formado por cardiologistas pediátricos e cirurgiões cardíacos pediátricos do Instituto do Coração de São Paulo [INCOR]), "DCC/CP" (grupo criado pelo Departamento de Cardiopatias Congênitas e Cardiologia Pediátrica da Sociedade Brasileira de Cardiologia, composto por cardiologistas pediátricos e cirurgiões cardíacos pediátricos de todo o país) e "GBCO-Ped" (grupo brasileiro de cardiooncologia pediátrica que inclui cardiologistas pediátricos com interesse em cardio-oncologia). Esses grupos incluem um total de mais de 350 indivíduos. Contatos pessoais do primeiro autor também foram convidados a participar. Um questionário com 10 perguntas (material suplementar) foi convertido em formato eletrônico via SurveyMonkey (Palo Alto, CA, EUA) e enviado aos participantes. Solicitou-se que os respondentes que trabalhassem em mais de uma instituição, respondessem ao questionário considerando apenas uma delas. A identidade dos respondentes e suas instituições foram preservadas, permitindo, assim a participação de vários respondentes de uma mesma instituição.

Devido ao grande número de contatos da cidade de São Paulo (SP), e como a cidade tem metade dos centros de cirurgia cardíaca do estado, com uma população maior do que a das regiões Norte e Centro-Oeste (figura 1), optamos por tratar a cidade de São Paulo como área geográfica separada, ao invés de considerá-la como parte da região Sudeste, para fins deste estudo. ${ }^{10}$

O questionário continha perguntas a respeito dos respondentes (local de trabalho, especialização e número de cirurgias cardíacas realizadas em sua principal instituição de referência), dos serviços de RMC (disponibilidade, especialista a frente dos exames e frequência dos exames), contexto clínico dos pacientes (indicação de RMC, disposição em indicar a operação de Fontan sem cateterismo cardíaco prévio, entre outros) e obstáculos enfrentados para utilizar a RMC nessa população. Questionários contendo apenas respostas sobre os respondentes foram excluídos das análises, já que essas informações sozinhas não teriam acrescentado dados relevantes aos objetivos do estudo. O mesmo questionário foi enviado a um grupo de WhatsApp formado por especialistas em imagem cardíaca pediátrica avançada do Brasil, do qual o autor principal faz parte, com o objetivo de obter dados sobre seus serviços de RMC.

Usamos as respostas à pergunta 3 (número de cirurgias realizadas anualmente) e à pergunta 6 (número de exames de RMC realizados mensalmente) para estimar a relação RMC/cirurgia para cada respondente. Transformamos todas as respostas categóricas individuais em números, já que as opções de resposta eram baseadas em números. Em relação ao número de cirurgias (pergunta 3), se menos de 150 cirurgias são realizadas anualmente, a resposta foi tratada como 100; se entre 150 e 249 cirurgias, a resposta foi tratada como 200; se entre 250 e 349 cirurgias, a resposta foi tratada como 300; e se mais de 350 cirurgias são realizadas por ano, a resposta foi tratada como 500. Em relação ao número de exames de RMC realizados mensalmente (pergunta 6), se nenhum exame de RMC é realizado, a respostas foi tratada como zero; se um ou dois exames de RMC são realizados, a resposta foi tratada como 2; se entre três e cinco exames, a resposta foi tratada como 5; se entre seis e doze exames RMC, a resposta foi tratada como 12; e se treze ou mais exames de RMC são realizados por mês, a resposta foi tratada como 20. Para transformar o índice de exames de RMC mensal em índice anual, os índices foram multiplicados por 12.

Usando esses números, pudemos estimar a relação RMC/ cirurgia para cada local, dividindo o número de exames de RMC pelo número de cirurgias cardíacas.

A complexidade da RMC foi estratificada como apresentado abaixo. Procedimentos de alta complexidade envolveram "CC complexa com dúvida diagnóstica", "pós-operatório de Jatene, 


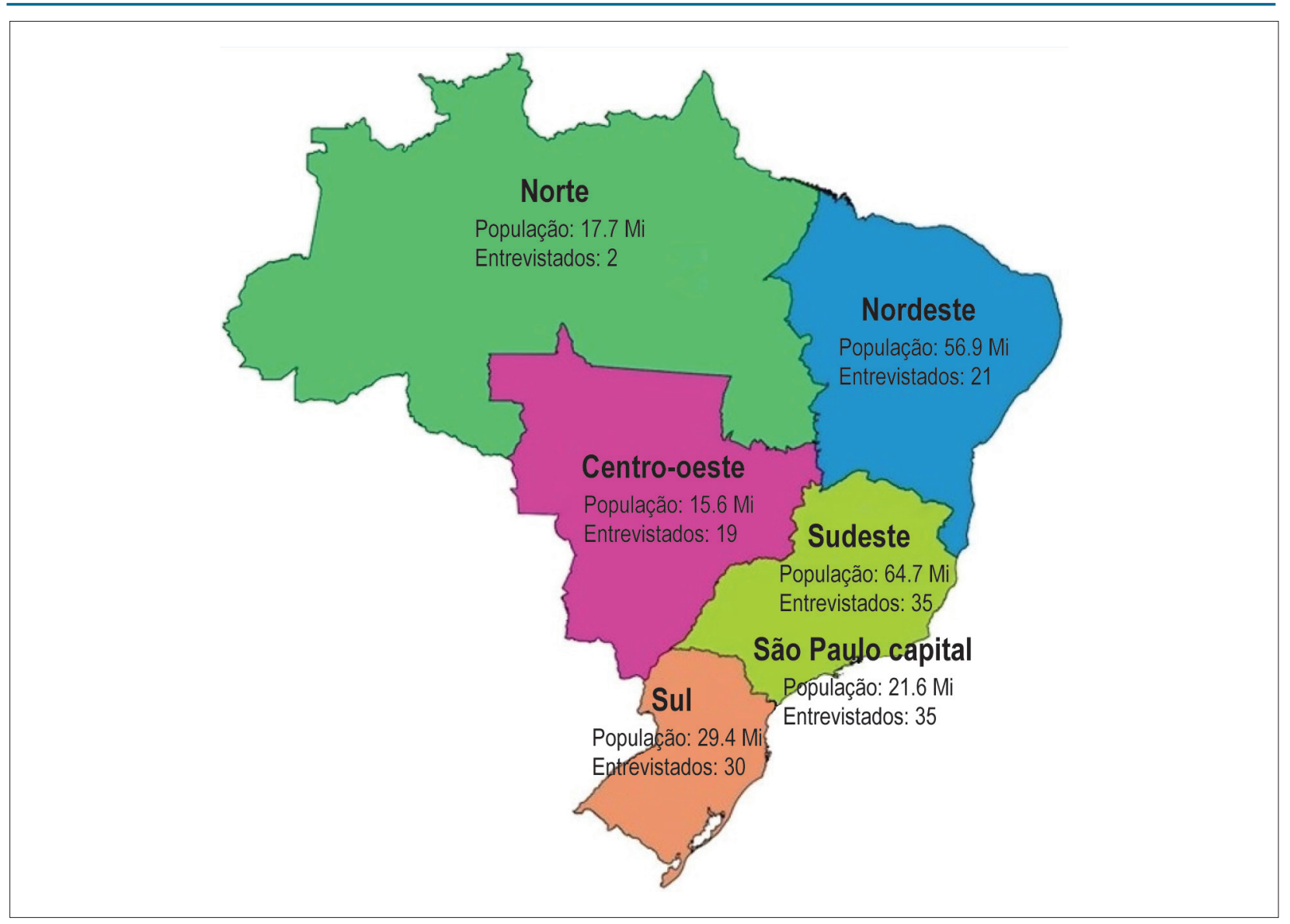

Figura 1 - Distribuição geográfica dos respondentes à pesquisa sobre RMC (adaptado de https://suportegeografico77.blogspot.com/2019/04/mapa-regioes-do-brasil.html).

Senning ou Mustard", "anomalia de Ebstein", "ventrículo direito ou esquerdo hipoplásico" (ventrículos limítrofes), "pré ou pós-operatório de Glenn e Fontan" e "RMC fetal". Procedimentos de média complexidade envolveram "pósoperatório de tetralogia de Fallot", "anomalias de situs" e "anomalia do retorno venoso pulmonar". Procedimentos de baixa complexidade incluíram todas as demais respostas. Essas indicações receberam uma classificação numérica: Alta complexidade $=3$, média complexidade $=2$ e baixa complexidade $=1$. As respostas individuais dos respondentes foram multiplicadas por essas pontuações somadas. A pontuação máxima possível era de 32 e cobria todas as indicações clínicas possíveis. Por exemplo: se alguém usa a RMC apenas para anomalia de Ebstein, ventrículo esquerdo hipoplásico, pós-operatório de tetralogia de Fallot e cardiomiopatia, a classificação de complexidade do serviço de RMC desse respondente é 9.

\section{Análise Estatística}

Considerando que o número de cardiologistas pediátricos registrados no Brasil era de 491 à época (portal.cfm.org.br), o tamanho da amostra estimada para obter um intervalo de confiança de 95\% (IC 95\%) com uma margem de erro de $\pm 3 \%$, de acordo com a fórmula de Cochran, era de 95 . Variáveis contínuas foram expressas como média \pm desvio padrão ou mediana com intervalo interquartil (25-75), conforme apropriado. Para a avaliação da normalidade dos dados, uma ferramenta de cálculo foi utilizada para calcular a área abaixo da curva normal, presumida como aquela onde aproximadamente 95\% da área está até 1,96 desvios-padrão da média. Variáveis categóricas foram apresentadas como números e porcentagens. O teste qui-quadrado foi utilizado para avaliar as associações entre variáveis categóricas e razões de chance (RC) ajustadas com seus IC 95\%. O teste de posto de Spearman foi utilizado para avaliar as correlações entre variáveis ordinais com distribuições distorcidas. Um $\mathrm{p}<0,05$ bilateral foi considerado estatisticamente significativo. As análises foram realizadas utilizando o programa StatsDirect, v. 2.7.2 2008 (Cheshire, Reino Unido).

\section{Resultados}

Nossa pesquisa obteve 142 respostas de um total de 364 entrevistados possíveis (índice de resposta 40\%). As respostas foram recebidas das seguintes regiões: Norte $(1,4 \%)$, Nordeste (14,8\%), Centro-Oeste (13,4\%), SP (24,65\%), Sudeste (não incluindo a cidade de São Paulo) $(24,65 \%)$ e Sul (21,1\%) (Figura 1). Devido à pouca participação de respondentes do Norte, essa região não foi representada em análises posteriores, que foram estratificadas por região. A maioria dos respondentes (75\%) trabalha nas capitais 
dos estados. A maioria foi composta de cardiologistas pediátricos (91,5\%), seguidos de cirurgiões cardíacos (7\%) e cardiologistas de adultos, que também atendem crianças $(1,4 \%)$. O tamanho dos programas de cirurgia cardíaca onde os médicos de referência trabalham variou de acordo com a região (Tabela 1 ).

Um total de $79 \%$ dos respondentes relatou que a RMC está 'disponível'. Esse índice variou amplamente de acordo com a região (Tabela 1), sendo que respondentes de Goiânia, Belém e Palmas relataram que a RMC não está disponível para eles. A RMC está disponível para $68 \%$ dos médicos que trabalham fora das capitais dos estados.

No Brasil, a RMC é realizada por radiologistas, seguida de cardiologistas pediátricos. Em algumas áreas, cardiologistas pediátricos a frente dos exames de RMC são raros ou inexistentes (Figura 2). A maioria dos respondentes (61\%) relatou que raramente ou nunca usam a RMC, enquanto 15\% relataram que a usam frequentemente. A frequência do uso de RMC variou por região geográfica (Tabela 1 ). A relação $\mathrm{RMC/}$ cirurgia no Brasil foi estimada entre 0,22 $\pm 0,27$, variando de acordo com a região (Tabela 1).

\section{Indicações de Ressonância Magnética Cardíaca}

Nossa pesquisa mostrou que as três indicações mais comuns para RMC são cardiomiopatias (84\%), pós-operatório de tetralogia de Fallot (81\%) e malformações do arco aórtico (53\%) (Tabela 2). Também foram encontradas diferenças regionais na complexidade da RMC (Tabela 1). Encontramos correlações entre a complexidade do exame e a relação RMC/ cirurgia (Rho $=0,48$, IC 95\% =0,32-0,62, p < 0,0001) e entre complexidade do exame e número de exames de RMC $($ Rho $=0,52$, IC 95\% $=0,38-0,64, p<0,0001)$. Nenhuma correlação foi encontrada entre a complexidade da RMC e o número de cirurgias $(p=0,73)$.

Tabela 1 - Resumo dos resultados mais importantes de pesquisa estratificados por região e a cidade de São Paulo

\begin{tabular}{|c|c|c|c|c|c|}
\hline & $\mathrm{NE}(\mathrm{n}=21)$ & $\operatorname{CO}(n=19)$ & SE $(n=35)$ & $\mathrm{SP}(\mathrm{n}=35)$ & $S(n=30)$ \\
\hline Número de cirurgias cardiacas estimado por centro/ano & $\begin{array}{c}200 \\
(200-300)\end{array}$ & $\begin{array}{c}180 \\
(180-180)\end{array}$ & $\begin{array}{c}200 \\
(100-307.5)\end{array}$ & $\begin{array}{c}400 \\
(200-500)\end{array}$ & $\begin{array}{c}300 \\
(200-480)\end{array}$ \\
\hline RMC disponivel - sim & $67 \%$ & $95 \%$ & $86 \%$ & $94 \%$ & $83 \%$ \\
\hline Cardiologista ped. realizando exames de RMC - sim & 0 & $68,5 \%$ & $28,2 \%$ & $68,6 \%$ & $30 \%$ \\
\hline Número de exames de RMC estimado por centro/mês (IQ) & $2(2-2)$ & $4,58(4,58-4,58)$ & $2(2-5)$ & $5(2-12)$ & $2(2-5)$ \\
\hline Classificação da complexidade da RMC: 0-32 (IQ) & $7(5-9)$ & $25(15-25)$ & $8(5,5-14)$ & $11(7-17,8)$ & $9(6-15)$ \\
\hline Razão RMC-cirurgia (DP) & $0,11 \pm 0,08$ & $0,35 \pm 0,27$ & $0,24 \pm 0,35$ & $0,36 \pm 0,35$ & $0,14 \pm 0,1$ \\
\hline
\end{tabular}

RMC: ressonância magnética cardíaca; Regiões NE: Nordeste; CO: Centro-oeste; SE: Sudeste; SP: São Paulo; S: Sul.

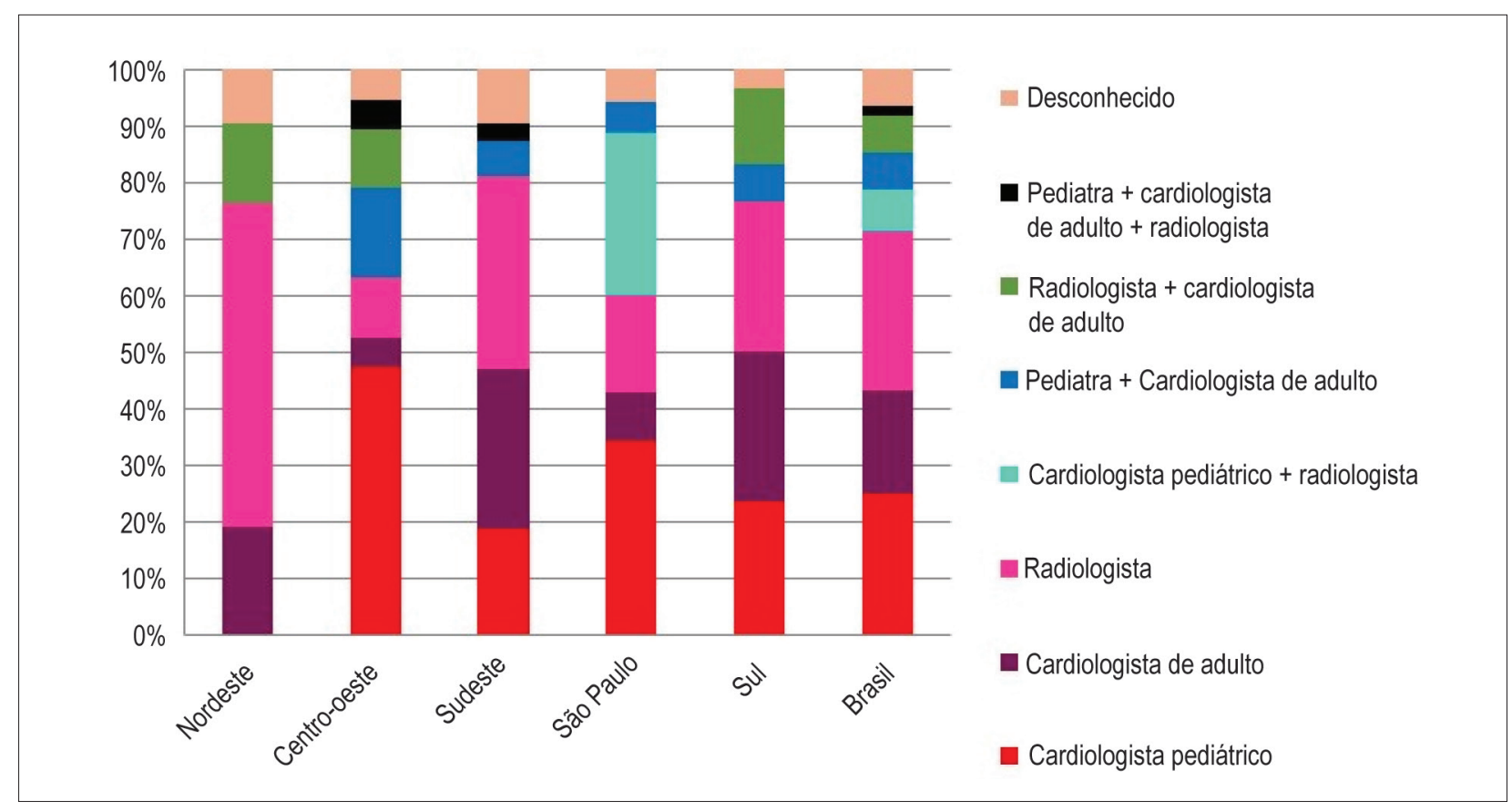

Figura 2 - Tipos de especialistas que realizam RMC pediátrica no Brasil de acordo com a região. Ped: pediatra 


\begin{tabular}{ll}
\hline \multicolumn{2}{l}{ Tabela 2 - Indicações de RMC para crianças no Brasil } \\
\hline Indicação de RMC & $\begin{array}{c}\text { Respostas } \\
\text { afirmativas (\%) }\end{array}$ \\
\hline Cardiomiopatias & 84 \\
\hline Status pós-reparo de tetralogia de Fallot & 81 \\
\hline Doença do arco aórtico & 53 \\
\hline DCC complexa & 46 \\
\hline Anomalia de Ebstein & 45 \\
\hline Tumores cardíacos & 44 \\
\hline Hipoplasia ventricular direita ou esquerda & 36 \\
\hline Pré- e/ou pós-Glenn e Fontan & 36 \\
\hline Status de pós-reparo de transposição das grandes artérias & 34 \\
\hline Anomalias coronárias & 32 \\
\hline Retorno venoso pulmonar & 31 \\
\hline Síndrome de Marfan & 18 \\
\hline Status pós-transplante cardíaco & 16 \\
\hline Cardiotoxicidade & 14 \\
\hline Anomalias de situs & 14 \\
\hline Arterite de Takayasu & 14 \\
\hline RMC fetal & 3 \\
\hline $\begin{array}{l}\text { RMC: ressonância } \\
\text { congênita. }\end{array}$ & cardíaca \\
\hline
\end{tabular}

Também encontramos uma correlação positiva entre alta complexidade da RMC $(\geq 18)$ e a presença de um cardiologista pediátrico a frente dos exames (RC 2,14, IC 95\% $1,2-3,89$, p 0,01 ), bem como uma correlação inversa entre alta complexidade e realização dos exames por um cardiologista de adultos (RC 0,42, IC 95\% 0,19-0,89, p 0,03).

\section{Necessidade de Cateterismo de Rotina Antes da Operação de Fontan}

Um número relativamente alto de respondentes (43\%) consideraria indicar operação de Fontan sem cateterismo cardíaco (CATE) prévio em pacientes selecionados, com base em achados do ecocardiograma (ECO) e da RMC.

Essa disposição em indicar a operação de Fontan sem CATE prévio está inversamente correlacionada ao tamanho do programa de cirurgia cardíaca (RC 0,6, IC 95\% 0,38-0,93, p $0,02)$ e à falta de confiança na avaliação de fluxos pela RMC (RC 0,42, IC 95\% 0,31-0,56, p < 0,0001).

\section{Confiança no Ecocardiograma e Cateterismo Cardíaco Versus RMC para Avaliação de Fluxos}

Entre os respondentes, $74 \%$ relataram confiar mais nas avaliações de fluxo por ECO e/ou CATE do que em avaliações de fluxo pela RMC.

Não identificamos associações entre confiança na avaliação de fluxos pela RMC, número de exames de RMC realizados mensalmente, uso de RMC para pré ou pós-operatório de Glenn e Fontan, ou tipo de especialistas à frente dos exames.

\section{Obstáculos ao Uso de RMC}

De acordo com os respondentes, os principais obstáculos ao uso mais frequente de RMC foram o alto custo (65\%), a necessidade de sedação (60\%) e um número insuficiente de profissionais qualificados (55\%; Tabela 3). Um dos respondentes mencionou que, em sua cidade, a RMC está disponível apenas para adultos.

\section{Discussão}

Até onde sabemos, esta é a primeira pesquisa a avaliar o uso de RMC em crianças no Brasil. Foram encontradas diferenças regionais em vários aspectos da prática com RMC: especialistas que realizam a RMC, indicações para $R M C$, relação $R M C$ / cirurgia, etc. Isso corrobora nossa impressão de que o uso de RMC é heterogêneo em todo o país. Este estudo identificou que, em comparação com os principais centros na Europa e América do Norte, a RMC não é suficientemente utilizada no Brasil, em relação ao número de cirurgias. Este estudo sugere que a razão para esse achado pode ser multifatorial.

O custo foi citado como o principal obstáculo ao uso mais frequente de RMC. Entretanto, considerando a disponibilidade relativamente alta de RMC pediátrica relatada nesta pesquisa, a resposta pode, por sua vez, refletir uma dificuldade de acesso a RMC. No Brasil, a maioria dos pacientes com cardiopatias congênitas são tratados em instituições públicas, que geralmente atuam com verbas muito restritas. Em evidente contraste com as necessidades de crianças e adolescentes com cardiopatias congênitas, de acordo com os dados de 2012 do Ministério da Saúde do Brasil, havia 84 aparelhos de ressonância magnética disponíveis para uso do Sistema Único de Saúde, e 1.263 aparelhos de ressonância magnética disponíveis para o sistema particular de saúde. A carência de aparelhos disponíveis é acentuada, ainda mais, pela necessidade de compartilhar o aparelho com outras especialidades. Na prática do autor principal, por exemplo, há apenas um aparelho disponível para exames de RMC pediátricos ou congênitos uma manhã por semana. Portanto, a relação RMC/cirurgia mais alta que pode ser atingida é de 0,6:1. Para fins de comparação, no Hospital for Sick Children em Toronto, há seis aparelhos para uso clínico, um

Tabela 3 - Obstáculos ao uso de RMC

\begin{tabular}{ll}
\hline Obstáculos & $\%$ \\
\hline Custo & 65 \\
\hline Necessidade de sedação & 60 \\
\hline Número insuficiente de profissionais qualificados & 55 \\
\hline Tempo de espera & 40 \\
\hline Conhecimento limitado e pouca promoção da RMC & 24 \\
\hline Inferioridade à angiografia por tomografia & 21 \\
computadorizada & 16 \\
\hline Credibilidade limitada & 5 \\
\hline Outros & \\
\hline
\end{tabular}

RMC: ressonância magnética cardíaca. 
deles dedicado exclusivamente a exames de RMC pediátrica por 6 horas diariamente. Outra explicação possível para os obstáculos da 'disponibilidade de aparelhos' é a questão do reembolso. Em nossa instituição, o sistema público de saúde reembolsa exames de RMC com R\$403, enquanto uma tomografia computadorizada (TC) é reembolsada com $\mathrm{R} \$ 322$, e um ECO é reembolsado com $\mathrm{R} \$ 165$. Em termos relativos, essas diferenças não são diferentes às existentes nos EUA. Portanto, essa não parece ser uma razão adequada para a subutilização da RMC em relação ao ECO e à TC. Em números absolutos, entretanto, o reembolso para todos os tipos de estudos de imagem é insuficiente. (http://www2. ebserh.gov. br/documents).

O segundo obstáculo mais importante a uma aplicação mais frequente da RMC foi identificado como sendo a necessidade de sedação ou anestesia, geralmente solicitada para crianças abaixo dos 8 anos de idade. Essa é uma preocupação frequente para os médicos dos pacientes e os pais das crianças. Entretanto, é importante enfatizar que o número de eventos adversos sofridos por crianças como resultado da sedação para RMC é muito baixo. ${ }^{11}$ Outro ponto a ser considerado é que a RMC é uma técnica não invasiva e que não expõe pacientes à radiação ionizante, caso do CATE cardíaco ou da TC. ${ }^{12}$ De qualquer forma, é importante mencionar que são tomadas precauções para evitar a sedação e que há muitas estratégias que podem ser adotadas para esse fim. ${ }^{13-16}$

O terceiro obstáculo mais importante ao uso mais frequente de RMC foi o número insuficiente de profissionais qualificados. A Sociedade de Ressonância Magnética Cardiovascular estratifica o treinamento para RMC em três níveis: 1. treinamento básico, 2. treinamento especializado e 3. treinamento avançado. ${ }^{17}$ Existe uma obrigatoriedade de se realizar pelo menos 150 exames de RMC por ano para obtenção do certificado de nível 2, que constitui o nível mínimo exigido na Europa para que cardiologistas pediátricos realizem RMC. ${ }^{17-19}$ No Brasil, não há regulamentação a esse respeito, mas não seria irracional buscar o padrão de referência europeu. Pode ser simplesmente uma questão de tempo até que a especialização em RMC pediátrica/congênita evolua em nosso país e que profissionais qualificados estejam disponíveis no mercado. É interessante observar que a força tarefa COCATS 4, reconhece que as habilidades em identificar cardiopatias congênitas simples e complexas em adultos, incluindo a quantificação de shunts cardíacos, geralmente são adquiridas após 36 meses de exposição aos casos de RMC em um serviço de RMC geral. ${ }^{20}$ Portanto, recomendase "concentrar" a experiência de aprendizagem fazendo o treinamento em instituições pediátricas especializadas. $\mathrm{Na}$ Europa e na América do Norte, por exemplo, são oferecidas bolsas para treinamento em RMC pediátrica/congênita em locais onde são realizados um alto número de exames pediátricos e congênitos. Os programas geralmente são localizados dentro de hospitais pediátricos, com um grande volume de cirurgias cardíacas. Muito frequentemente, esses locais têm cardiologistas pediátricos como membros integrantes de suas equipes de exames de imagem. No Brasil, esse cenário existe apenas na cidade de São Paulo, onde o número de cirurgias é alto e onde há cardiologistas pediátricos rotineiramente envolvidos na prática de RMC pediátrica. No momento atual, portanto, nos parece que o treinamento de radiologistas e cardiologistas para se tornarem especialistas em exames de RMC pediátricos, deveria envolver algum treinamento em centros acadêmicos na Europa e na América do Norte.

Quase todos os respondentes já solicitaram exames de RMC para pacientes com cardiomiopatia ou pósoperatório de tetralogia de Fallot, indicações de baixa e média complexidade. Exames mais complexos são realizados em centros com as relações RMC/cirurgia mais altas, especialmente quando cardiologistas pediátricos estão envolvidos nos exames. Por outro lado, não se identificou necessariamente a relação entre a complexidade dos exames e o número de cirurgias realizadas. É provável que locais que ofereçam RMC guiadas por cardiologistas pediátricos atraiam os casos mais complexos, talvez por estarem mais preparados para aceitar esses casos, ou por esses serviços estarem mais integrados à prática cardiológica.

$\mathrm{Na}$ ampla maioria dos centros mundiais, é rotina que pacientes com coração univentricular passem por um CATE cardíaco pré-Fontan para avaliar a anatomia arterial pulmonar, medir a pressão do leito arterial pulmonar e estimar a resistência vascular pulmonar. Alguns especialistas têm encorajado o uso apenas de RMC nesses casos, sendo o CATE destinado apenas a pacientes de alto risco. ${ }^{21-25}$ Nessa pesquisa, $43 \%$ dos respondentes relataram que estariam dispostos a indicar uma operação de Fontan apenas com informações de ECO e RMC, evitando, assim, o CATE em pacientes considerados de risco padrão, embora tenhamos observado uma grande variação nas práticas entre regiões.

\section{Limitações do Estudo}

Reconhecemos que, embora o tamanho da amostra estimada seja suficiente para responder nossas perguntas, o alto número de não respondentes, algo esperado nesse tipo de pesquisa, é uma fonte de viés em potencial. A limitação mais importante da pesquisa, entretanto, foi o fato de que o número real de exames de RMC pediátricas por centro continua desconhecido. Tentamos obter esses números de radiologistas e cardiologistas que são responsáveis ou fazem parte de equipes de imagem dos maiores programas de cirurgia cardíaca pediátrica no Brasil, mas apenas uma instituição concordou em compartilhar seus dados. Portanto, as relações $\mathrm{RMC}$ /cirurgia encontradas, e todas as associações entre o número de exames de RMC e número de cirurgias foram simples estimativas, e podem não ser, até certo ponto, representativas da situação no Brasil. Para mitigar incertezas e melhorar a comparabilidade entre instituições, oferecemos opções de resposta que incluíram faixas de números ao invés de números precisos. É possível que tenhamos recebido respostas de vários indivíduos da mesma instituição e que essas respostas possam, até certo ponto, ter expressado estimativas individuais e não dados institucionais concretos, e, também, que algumas instituições possam ter sido desproporcionalmente mais representadas no grupo de respondentes. Da mesma forma, não conseguimos avaliar a disponibilidade da RMC e outros aspectos na região Norte do Brasil, devido à baixa participação de colegas dessa área. 


\section{Conclusões}

A RMC pediátrica está disponível para aproximadamente 2/3 dos médicos, mas não é muito frequentemente utilizada no Brasil. Os exames geralmente são realizados por radiologistas. A RMC é mais comumente indicada para cardiomiopatias e pós-operatório de tetralogia de Fallot. A presença de um cardiologista pediátrico a frente dos exames de RMC está associada ao uso de RMC em pacientes mais complexos. Os obstáculos ao uso mais frequente da RMC foram custo, necessidade de sedação e falta de profissionais qualificados. Um maior treinamento de especialistas em RMC pediátrica e uma maior educação sobre o método aos médicos solicitadores dos exames são passos importantes para o aumento do uso de RMC no Brasil. A colaboração entre instituições é recomendada e necessária a fim de se ter um cenário mais fidedigno sobre o uso da RMC na população pediátrica no Brasil.

\section{Agradecimentos}

Gostaríamos de agradecer aos colegas que contribuíram com seu tempo para responder à pesquisa. Gostaríamos de agradecer, especialmente, aos Drs. Antônio Soares Souza e Mari Roedo, radiologistas do Hospital de Base de São José do Rio Preto, por terem compartilhado conosco o número de exames de RMC de sua instituição.

\section{Referências}

1. Hendel RC, Patel MR, Kramer CM, Poon M, Carr JC, Gerstad NA, et al. ACCF/ ACR/SCCT/SCMR/ASNC/NASCI/SCAI/SIR 2006 Appropriateness Criteria for Cardiac Computed Tomography and Cardiac Magnetic Resonance Imaging: A Report of the American College of Cardiology Foundation Quality Strategic Directions Committee Appropriateness Criteria Working Group, American College of Radiology, Society of Cardiovascular Computed Tomography, Society for Cardiovascular Magnetic Resonance, American Society of Nuclear Cardiology, North American Society for Cardiac Imaging, Society for Cardiovascular Angiography and Interventions, and Society of Interventional Radiology. J Am Coll Cardiol. 2006;48(7):1475-97.

2. Clarke CJ, Gurka MJ, Norton PT, Kramer CM, Hoyer AW. Assessment of the accuracy and reproducibility of RV volume measurements by CMR in congenital heart disease. JACC Cardiovasc Imaging. 2012;5(1):28-37.

3. Hundley WG, Li HF, Hillis LD, Meshack BM, Lange RA, Willard JE, et al. Quantitation of cardiac output with velocity-encoded, phase-difference magnetic resonance imaging. Am J Cardiol. 1995;75(17):1250-5.

4. Hundley WG, Li HF, Lange RA, Pfeifer DP, Meshack BM, Willard JE, et al. Assessment of left-to-right intracardiac shunting by velocity-encoded, phase difference magnetic resonance imaging. A comparison with oximetric and indicator dilution techniques. Circulation. 1995;91(12):2955-60.

5. Powell AJ, Maier SE, Chung T, Geva T. Phase-velocity cine magnetic resonance imaging measurement of pulsatile blood flow in children and young adults: in vitro and in vivo validation. Pediatr Cardiol. 2000;21(2):104-10

6. Mewton N, Liu CH, Croisille P, Bluemke D, Lima JAC. Assessment of myocardial fibrosis with cardiovascular magnetic resonance. J Am Coll Cardiol. 2011:57(8):891-903.

7. Jordan JH, Vasu S, Morgan TM, D'Agostino Jr RB, Melendez GC, Hamilton CA, et al. Anthracycline-associated T1 mapping characteristics are elevated independent of the presence of cardiovascular morbidities in cancer survivors. Circ Cardiovasc Imaging. 2016;9(8):e004325.

\section{Contribuição dos Autores}

Concepção e desenho da pesquisa, obtenção de dados e análise estatística: Kozak MF. Análise e interpretação dos dados, redação do manuscrito e revisão crítica do manuscrito quanto ao conteúdo intelectual importante: Kozak MF, Afiune JY, Grosse-Wortmann L.

\section{Potencial Conflito de Interesses}

Declaramos não haver conflito de interesses pertinentes.

\section{Fontes de Financiamento}

O presente estudo não teve fontes de financiamento externas.

\section{Vinculação Acadêmica}

Não há vinculação deste estudo a programas de pósgraduação.

\section{Aprovação Ética e Consentimento Informado}

Este artigo não contém estudos com humanos ou animais realizados por nenhum dos autores.

8. Costello JP, Olivieri LJ, Su L, Krieger A, Alfares F, Thabit O, et al. Incorporating three-dimensional printing into a simulation-based congenital heart disease and critical care training curriculum for resident physicians. Congenit Heart Dis. $2015 ; 10(2): 185-90$

9. Costello JP, Olivieri LJ, Krieger A, Thabit O, Marshall MB, Yoo SJ, et al. Utilizing three-dimensional printing technology to assess the feasibility of high-fidelity synthetic ventricular septal models for simulation in medical education. World J Pediatr Congenit Heart Surg. 2014;5(3):421-6.

10. Caneo LF, Jatene MB, Yatsuda N, Gomes WJ. A reflection on the performance of pediatric cardiac in the State of São Paulo. Rev Bras Cir Cardiovasc. $2012 ; 27(3): 457-62$

11. Rangamani S, Varghese J, Li L, Harvey L, Hammel JM, Fletcher SE, et al. Safety of cardiac magnetic resonance and contrast angiography for neonates and small infants: a 10-year single-institution experience. Pediatr Radiol. 2012;42(11):1339-46.

12. Callahan MJ, MacDougall RD, Bixby SD, Voss SD, Robertson RL, Cravero JP. Ionizing radiation from computed tomography versus anesthesia for magnetic resonance imaging in infants and children: patient safety considerations. Pediatr Radiol. 2018;48(1):21-30.

13. Kharabish A, Mkrtchyan N, Meierhofer C, Martinoff S, Ewert P, Stern H, et al. Cardiovascular magnetic resonance is successfully feasible in many patients aged 3 to 8 years without general anesthesia or sedation. J Clin Anesth. 2016 Nov; $34: 11-4$

14. Lu JC, Nielsen JC, Morowitz L, Musani M, Mahani MG, Agarwal PP, et al Use of a 1.0 Tesla open scanner for evaluation of pediatric and congenital heart disease: a retrospective cohort study. J Cardiovasc Magn Reson. 2015;17(1):39.

15. Shariat M, Mertens L, Seed M, Grosse-Wortmann L, Golding F, MarcerRosa L, et al. Utility of feed-and-sleep cardiovascular magnetic resonance in young infants with complex cardiovascular disease. Pediatr Cardiol. 2015;36(4):809-12. 


\section{Artigo Original}

16. Windram J, Grosse-Wortmann L, Shariat M, Greer ML, Crawford CW, Yoo SJ. Cardiovascular MRI without sedation or general anesthesia using a feed-andsleep technique in neonates and infants. Pediatr Radiol. 2012;42(2):183-7.

17. Kim RJ, Simonetti OP, Westwood M, Kramer CM, Narang A, Friedrich MG et al. Guidelines for training in cardiovascular magnetic resonance (CMR). J Cardiovas Mag Reson. 2018;20:57.

18. Helbing WA, Mertens L, Sieverding L. Recommendations from the Association for European Paediatric Cardiology for training in congenital cardiovascular magnetic resonance imaging. Cardiol Young. $2006 ; 16(4): 410-2$

19. Plein S, Schulz-Menger J, Almeida A, Mahrholdt H, Rademakers F, Pennell $\mathrm{D}$, et al. Training and accreditation in cardiovascular magnetic resonance in Europe: a position statement of the working group on cardiovascular magnetic resonance of the European Society of Cardiology. Eur Heart J. 2011;32(7):793-8.

20. Kramer CM, Hundley WG, Kwong RY, Martinez CW, Raman SV, Ward RP. COCATS 4 task force 8: training in cardiovascular magnetic resonance imaging. J Am Coll Cardiol. 2015;65(17):1822-31.
21. Downing TE, Whitehead KK, Dori Y, Gillespie MJ, Harris MA, Fogel MA, et al. Accuracy of conventional oximetry for flow estimation in patients with Superior cavopulmonary connection: a comparison with phase-contrast MIR. Circ Cardiovasc Imaging. 2013;6(6):943-9.

22. Fogel MA, Pawlowski TW, Whitehead KK, Harris MA, Keller MS, Glatz AC, et al. Cardiac magnetic resonance and the need for routine cardiac catheterization in single ventricle patients prior to fontan: a comparison of 3 groups. J Am Coll Cardiol. 2012;60(12):1094-1102

23. Prakash A, Khan MA, Hardy R, Torres AJ, Chen JM, Gersony WM. A new diagnostic algorithm for assessment of patients with single ventricle before a Fontan operation. J Thorac Cardiovasc Surg. 2009;138(4):917-23.

24. Ven JPG, Bosch E, Bogers AJCC, Helbing WA. State of the art of the Fontan strategy for treatment of univentricular heart disease. F1000Res. 2018 Jun $27 ; 7: F 1000$.

25. Brown DW, Gauvreau K, Powell AJ, Lang P, Nido PJ, Odegard KC, et al. Cardiac magnetic resonance versus routine cardiac catheterization before bidirectional Glenn anastomosis: long-term follow-up of a prospective randomized trial. J Thorac Cardiovasc Surg. 2013;146(5):1172-8. 